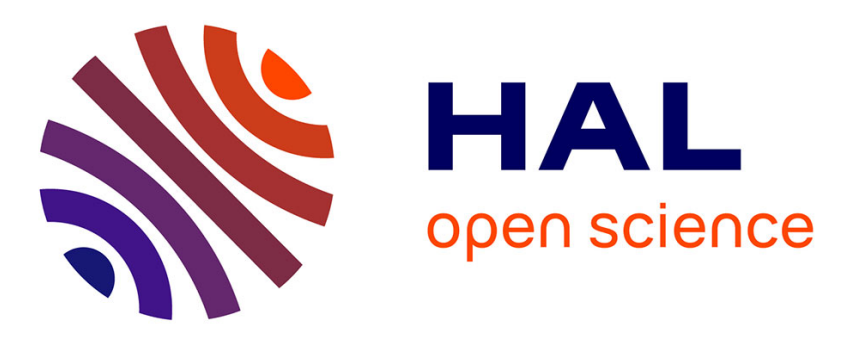

\title{
Identification of photon-tagged jets in the ALICE experiment
}

\author{
G. Conesa, H. Delagrange, J. Diaz, Y.V. Kharlov, Y. Schutz
}

\section{To cite this version:}

G. Conesa, H. Delagrange, J. Diaz, Y.V. Kharlov, Y. Schutz. Identification of photon-tagged jets in the ALICE experiment. Nuclear Instruments and Methods in Physics Research Section A: Accelerators, Spectrometers, Detectors and Associated Equipment, 2008, 585, pp.28-39. 10.1016/j.nima.2007.10.050 . in2p3-00188008

\section{HAL Id: in2p3-00188008 https://hal.in2p3.fr/in2p3-00188008}

Submitted on 15 Nov 2007

HAL is a multi-disciplinary open access archive for the deposit and dissemination of scientific research documents, whether they are published or not. The documents may come from teaching and research institutions in France or abroad, or from public or private research centers.
L'archive ouverte pluridisciplinaire HAL, est destinée au dépôt et à la diffusion de documents scientifiques de niveau recherche, publiés ou non, émanant des établissements d'enseignement et de recherche français ou étrangers, des laboratoires publics ou privés. 


\title{
Identification of Photon-tagged Jets in the ALICE Experiment
}

\author{
G. Conesa ${ }^{\mathrm{a}, \mathrm{b}, *}$ H. Delagrange ${ }^{\mathrm{b}}$ J. Díaz $^{\mathrm{a}}$ Y.V. Kharlov ${ }^{\mathrm{c}}$ \\ Y. Schutz ${ }^{b, d}$ \\ ${ }^{a}$ IFIC (Centro Mixto Universidad de Valencia-CSIC), Valencia, Spain \\ ${ }^{\mathrm{b}}$ SUBATECH, Ecole des Mines, IN2P3/CNRS, Université de Nantes, Nantes,
}

France

${ }^{\mathrm{c} I n s t i t u t e ~ f o r ~ H i g h-E n e r g y ~ P h y s i c s, ~ P r o t v i n o, ~ R u s s i a ~}$

${ }^{\mathrm{d}}$ CERN, Genève, Switzerland

\begin{abstract}
The ALICE experiment at LHC will detect and identify prompt photons and light neutral-mesons with the PHOS detector and the additional EMCal electromagnetic calorimeter. Charged particles will be detected and identified by the central tracking system. In this article, the possibility of studying the interaction of jets with the nuclear medium, using prompt photons as a tool to tag jets, is investigated by simulations. New methods to identify prompt photon-jet events and to distinguish them from the jet-jet background are presented.
\end{abstract}

Key words: High-energy gamma rays, electromagnetic calorimeters, quark-gluon plasma.

PACS: 25.75.Nq, 24.10.Lx, 25.75.-q, 29.40.Vj 


\section{Introduction}

In Ref. [1], we stressed the importance of the measurement of jets in the ALICE experiment at LHC to study the properties of the nuclear medium predicted to be created in ultra-relativistic heavy-ion collisions, the QuarkGluon Plasma [2,3]. Jets will be abundantly produced at the LHC $\left(2 \times 10^{6}\right.$ jets with $p_{T}>100 \mathrm{GeV} / c$ per year in the ALICE acceptance) enabling inclusive and exclusive jet measurements. In particular, jet topology (jet shape, jet heating, fragmentation function,... ) can be measured to study the redistribution of the jet energy among the fragmentation particles after the jet has traversed the nuclear medium created in the collision [4]. These studies require the identification of jets and the measurement, as accurately as possible, of the jet energy, ideally before and after interaction with the medium. A very attractive method to perform these studies is to tag jets with prompt photons emitted opposite to the jet direction. The dominant processes for such events are $g+q \rightarrow \gamma+q$ (Compton) and $q+\bar{q} \rightarrow \gamma+g$ (annihilation), although recent studies show that Next to Leading Order processes (NLO) contribute significantly to the photon spectrum below $50 \mathrm{GeV} / c[5]$. As photons emerge almost unaltered from the dense medium, they provide a measurement of the original energy of the parton emitted in the opposite direction. On one hand, this coincidence technique can be used to localize the jet and on the other hand it allows to build the parton fragmentation function without the need of reconstructing the jet energy from the detected hadrons. Medium effects can then be identified through modifications of the fragmentation function, i.e., by the redistribution of the jet energy among its components.

\footnotetext{
* Corresponding author

Email address: Gustavo. Conesa.Balbastre@cern.ch (G. Conesa).
} 
The identification of prompt photons in ALICE with the high resolution photon spectrometer PHOS in association with the central tracking system has been presented earlier $[1,6]$. The identification of prompt-photon jet events in ALICE is found optimal for photons with energy larger than $20 \mathrm{GeV}$. Below this energy, decay and prompt photons cannot be efficiently separated on an event by event basis.

In this paper, we discuss the possibilities offered by the addition of the EMCal electromagnetic calorimeter to the ALICE setup. We present an algorithm for identifying photon-jet events and for reconstructing hadron jet features. The algorithm was developed and tested with the simulations described in Ref. [1]. The study was done with generated particles reconstructed with a fast reconstruction algorithm, due to lack of computer time for a full reconstruction. The generator used to produce $p p$ collisions at $\sqrt{s}=5.5 \mathrm{TeV}$ is PYTHIA $6.203[7,8]$. The simulations generated with PYTHIA contain $\gamma$-jet and jetjet events, with prompt and decay photons, respectively, in the energy range $20 ; E_{j} 100 \mathrm{GeV}$. The generator used to reproduce the underlying event of a heavy-ion collision is HIJING 1.36 [9]. To show the improvements provided by the electromagnetic calorimeter EMCal in the jet reconstruction procedure, two possible experimental situations are considered: i) only charged particles can be detected (EMCal is not present) and ii) neutral particles can also be detected (EMCal is present).

\section{Acceptance and response of the detectors}

To assess the performance of our photon-jet identification algorithm, a fast detector simulation is used because a complete event simulation with particle 


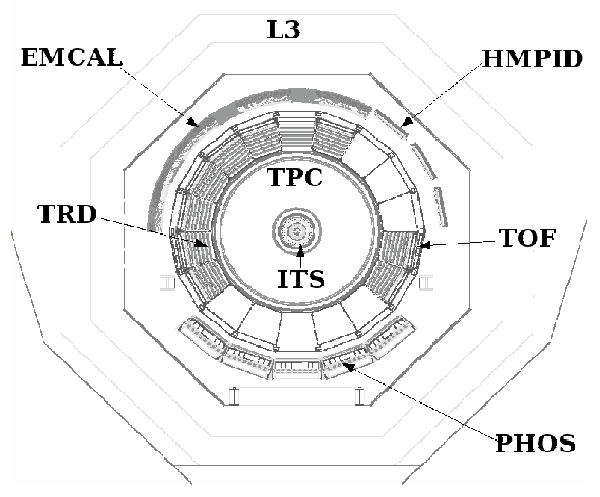

Fig. 1. Sketch of the ALICE experiment, transverse view.

transport and reconstruction would have required an unaffordable computing time. In this fast simulation framework, we rely only on the properties of the final state particles generated by PYTHIA and on the knowledge of the response function and acceptance of the various detectors involved.

The ALICE experiment is sketched in Fig. 1. The acceptances of all the detectors relevant for this study are listed in Table 1. A full description of the ALICE detector can be found in Ref. [10].

The response function of the detectors involved in our analysis, PHOS and the central tracking system (TPC), are described in Sec. 3 of Ref. [1]. The EMCal response was assumed to be identical to the PHOS response, although in fact it will be slightly worse. However, since the EMCAL granularity is coarser, the $p_{T}$ range over which a Shower Shape Analysis (SSA) can discriminate prompt photons from decay photons ${ }^{1}$ is reduced from about $100 \mathrm{GeV} / c$ in PHOS down to $40 \mathrm{GeV} / c$ in EMCal.

$\overline{1}$ Due to Lorentz contraction and the calorimeters granularity decay photons merge in one detected cluster for $E_{\pi^{0}} \gtrsim 30 \mathrm{GeV}$ in PHOS and $E_{\pi^{0}} \gtrsim 10 \mathrm{GeV}$ in EMCal. 
Table 1

TPC (Time Projection Chamber), PHOS and EMCal acceptances. The physical TPC $\eta$ acceptance is larger $(|\eta|<0.9)$, but we take this lower value to ensure a good track matching. The acceptance of EMCal used in this study might not be the final one as the layout is still under discussion.

\begin{tabular}{cccc} 
Detector & $|\eta|$ & $\phi_{\min }$ & $\phi_{\max }$ \\
\hline \hline PHOS & 0.12 & $220^{\circ}$ & $320^{\circ}$ \\
EMCAL & 0.7 & $60^{\circ}$ & $180^{\circ}$ \\
TPC & 0.7 & $0^{\circ}$ & $360^{\circ}$ \\
\hline
\end{tabular}

\section{Jet selection procedure}

Photon-jet events were identified by applying the following $\gamma$-tagging algorithm:

(1) Find the most energetic prompt photon identified in PHOS (Ref. [1]).

(2) Find the jet leading particle ${ }^{2}$, either a charged hadron detected by the central tracking system or a neutral pion detected by the EMCal, with the highest $p_{T}$ value and emitted back-to-back to the photon $(\Delta \phi \sim$ $180^{\circ}$ ). Neutral pions are identified from their two photon decay from all photons in the event with a relative angle between the limits defined by the decay kinematics and with an invariant mass in the range $120<$ $M_{\gamma \gamma}<150 \mathrm{MeV} / c^{2}$. In addition, it is required that the $p_{T}$ value of the leading particle be at least $10 \%$ of the photon energy.

(3) Reconstruct the jet as the ensemble of all particles above a given $p_{T}$

$\overline{2}$ Jets always have a particle carrying a significant fraction of the jet energy (in average $40 \%)$. 
threshold contained inside a cone of a given radius around the leading particle direction.

(4) Finally, the event is identified as a photon-jet if the ratio of the reconstructed jet energy and the prompt photon energy falls within a given selection window.

There are standard algorithms that can be used to reconstruct jets $\left(k_{T}\right.$, cone, etc), but they fail to reconstruct jets with energy smaller than $40-50 \mathrm{GeV}$ in a heavy-ion environment $[11,6]$. Studies about tagging reconstructed jets with prompt photons with standard algorithms are in progress.

\subsection{Leading particle selection}

In ALICE, since the central tracking system and EMCal do not cover the same acceptance, jets can be fully reconstructed (charged plus neutral hadrons) only over a limited solid angle $\left(|\eta|<0.7\right.$ and $\Delta \phi=120^{\circ}$ in this study $\left.{ }^{3}\right)$. Therefore, outside the common acceptance region, the jet energy is only partially reconstructed which may lead to the rejection of a true $\gamma$-jet event.

The prompt photon and the parton at the origin of the observable $\gamma$-jet event are emitted in opposite directions in the center of mass of the hard process. In the laboratory system, the correlation in pseudo-rapidity is washed out, as illustrated in Fig. 2, while the azimuthal angle correlation is conserved since there is no boost in the transverse direction. The relative azimuthal angle, $\Delta \phi$, of $\gamma$-jet events is peaked at $180^{\circ}$ and its width depends on both the

3 The EMCal design is still under discussion and now, an azimuthal aperture of $110^{\circ}$ is considered. 

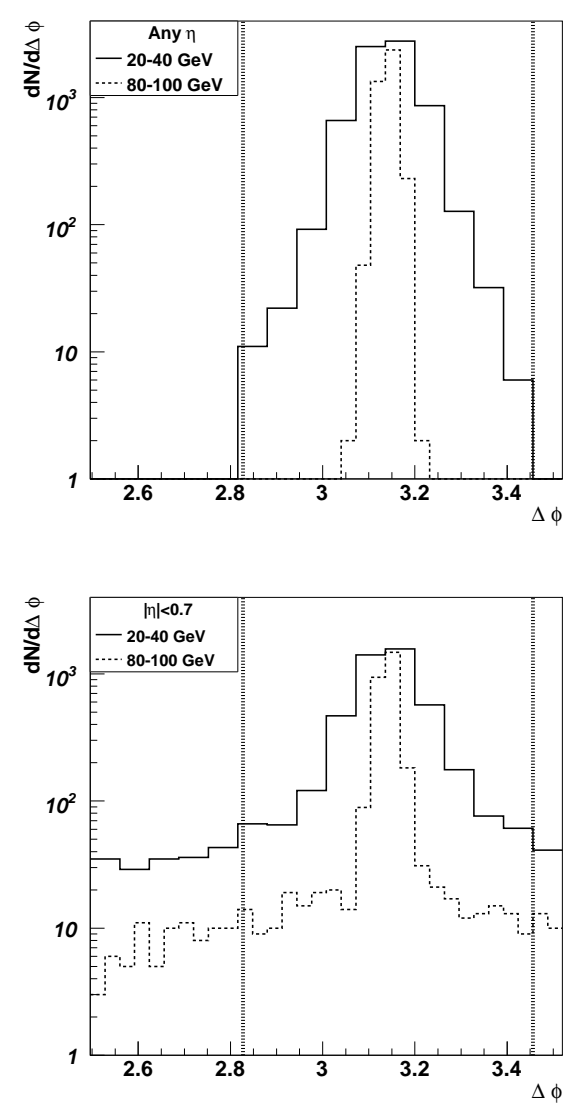

Fig. 2. Azimuthal angle correlations between prompt photons and their corresponding jet leading particles as a function of $\Delta \phi=\phi_{l}-\phi_{\gamma}$ for simulated $\gamma$-jet events in the energy ranges $20-40 \mathrm{GeV}$ and $80-100 \mathrm{GeV}$ for $p p$ collisions. Jet particles (lower frame) are filtered through the acceptance $|\eta|<0.7$. Dotted vertical lines represent the cut between $0.9 \pi$ and $1.1 \pi$ that we use to select leading particles.

detector acceptance and resolution, and the energy of the event. We have tuned our algorithm to select photon leading-particle pairs satisfying the angular condition $0.9 \pi<\Delta \phi<1.1 \pi$.

When the leading particle of a given event escapes from the detector acceptance, the algorithm finds a wrong leading particle. This misidentification produces the peak in the jet leading particle distribution at low values of the $p_{T, l} / E_{\gamma}$ ratio (Fig. 3), where $p_{T, l}$ and $E_{\gamma}$ are the momentum of the identified 


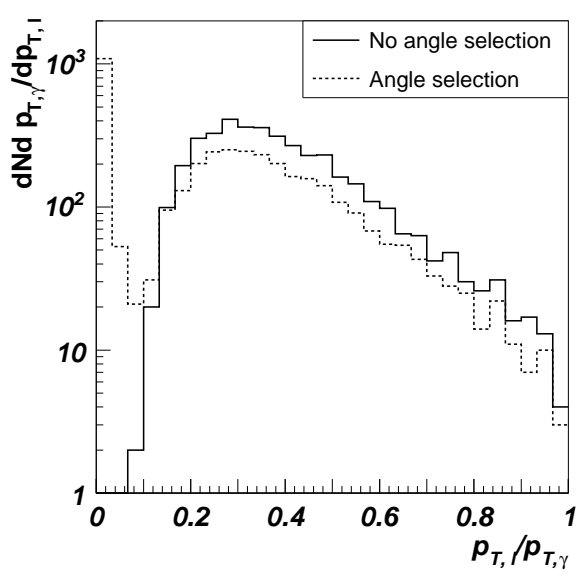

Fig. 3. Leading particle distribution of $\gamma$-jet events as a function of the $p_{T, l} / E_{\gamma}$ ratio for $p p$ collisions and jet energies in the range $80-100 \mathrm{GeV}$. The dotted line corresponds to the case where the leading particle is searched inside the acceptance of the detectors and opposite in $\phi$ angle to the prompt photon. The solid line corresponds to the case whithout acceptance restrictions.

leading particle and the energy of the photon, respectively. These fake leading particles are rejected by imposing the condition $p_{T, l} / E_{\gamma}>0.1$. The probability of finding the jet associated to a prompt photon is about $50 \%$, determined only by the jet detection acceptance.

\subsection{Identification of $\pi^{0}$ leading particles}

The $\pi^{0}$ candidates to leading particles are identified by detecting their two decay photons in EMCal and selecting those with an invariant mass $M_{\gamma \gamma}$ around the $\pi^{0}$ rest mass. Because of the combinatorial background, which is particularly large in the case of $\mathrm{Pb}-\mathrm{Pb}$ collisions (Fig. 4), additional selection conditions are necessary.

The opening angle $\theta_{12}$ between the two $\pi^{0}$ decay photons is given in the lab 


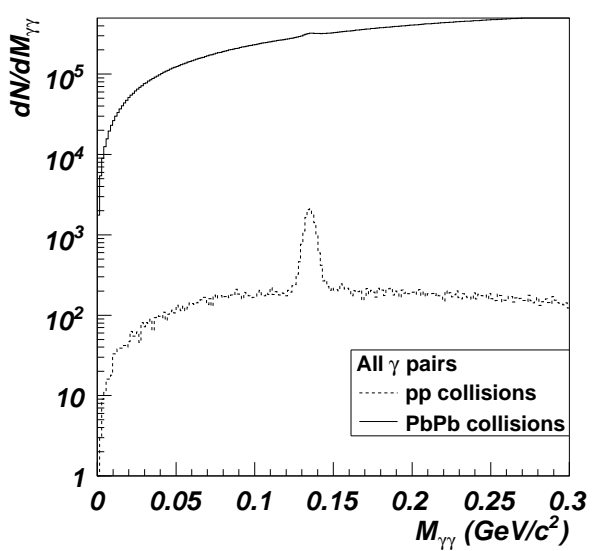

Fig. 4. Invariant mass distributions of all photon pairs in the event for $p p$ (dashed line) and $\mathrm{Pb}-\mathrm{Pb}$ (solid line) collisions at $\sqrt{s}=5.5 \mathrm{~A} \mathrm{TeV}$. No selection is applied.

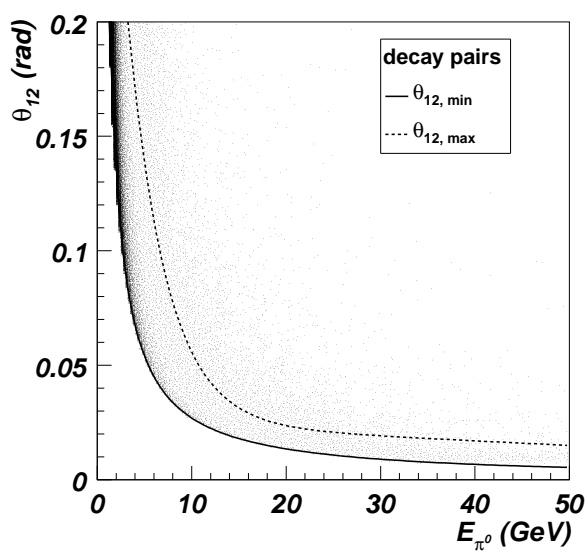

Fig. 5. Opening angle of $\pi^{0}$ decay photon pairs (without any restriction in detector acceptance). The lines limit the opening angle range selected; the lower line is obtained from the decay kinematics, the upper one has been empirically chosen to select most of $\pi^{0}$ decay pairs.

system by:

$$
\cos \theta_{12}=\frac{\gamma_{\pi}^{2} \beta_{\pi}^{2}-\gamma_{\pi}^{2} \alpha^{2}-1}{\gamma_{\pi}^{2}\left(1-\alpha^{2}\right)}
$$

where $\alpha$ is the decay asymmetry, $\gamma$, the Lorentz factor and $\beta$ the $\pi^{0}$ velocity in units of $c$. The opening angle is minimum for symmetric decays $(\alpha=0)$. 

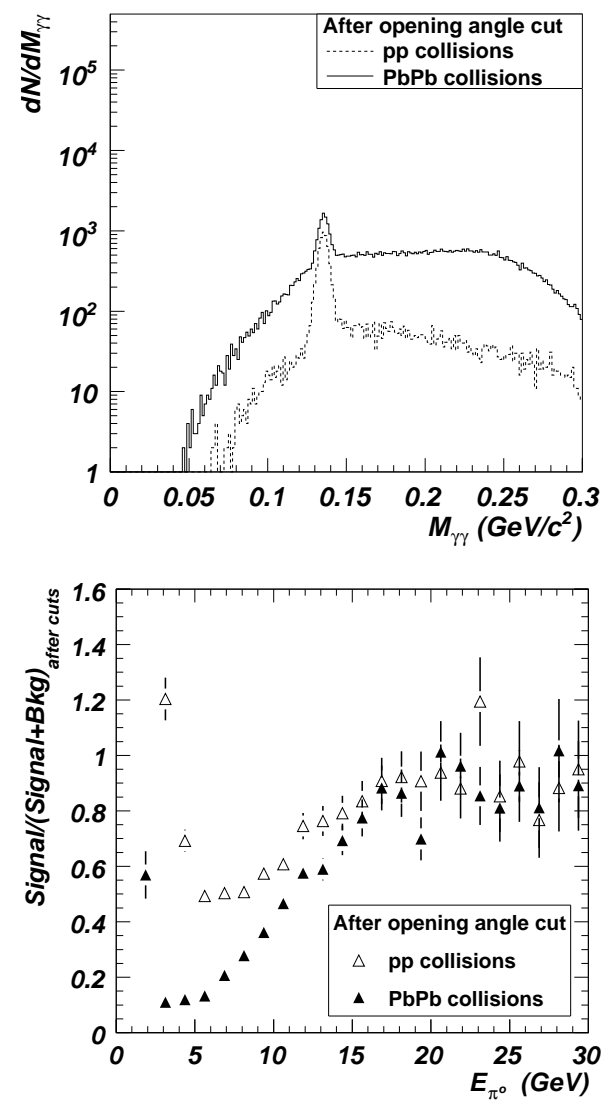

Fig. 6. Upper frame: Invariant mass distributions of photon pairs in the event which satisfy the $\phi$ and leading particle energy cuts and the opening angle restriction, for $p p$ (dashed line) and $\mathrm{Pb}-\mathrm{Pb}$ (solid line) collisions at $\sqrt{s}=5.5 \mathrm{~A} \mathrm{TeV}$. Lower frame: Ratio of the simulated $\pi^{0}$ number to the selected photon pair number in the event, for $p p(\triangle)$ and $\mathrm{Pb}-\mathrm{Pb}(\boldsymbol{\Lambda})$ collisions at $\sqrt{s}=5.5 A \mathrm{TeV}$. Only $\gamma$-jet events with jet energy in the range from 20 to $100 \mathrm{GeV}$ are considered.

From the simulated $\pi^{0}$ opening angle distributions in $\mathrm{Pb}-\mathrm{Pb}$ collisions (Fig. 5), the maximum opening angle was empirically selected by fitting the equation

$$
\theta_{\max }=0.4 \cdot e^{-0.25 \cdot E}+0.025-2 \cdot 10^{-4} \cdot E_{\pi^{o}} .
$$

to the data.

The condition $\theta \leq \theta_{\max }$ selects more than $80 \%$ of the $\pi^{0}$ decay photon pairs ${ }^{4}$ $\overline{4}$ The selection efficiency decreases from about $92 \%$ at $E_{\pi} \sim 2 \mathrm{GeV}$ to around 


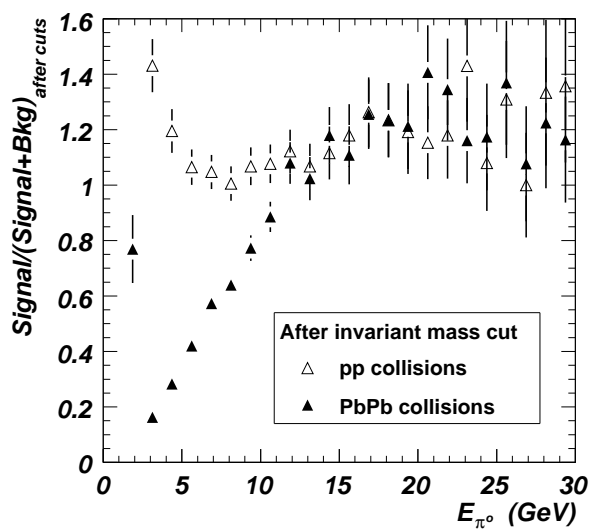

Fig. 7. Ratio of number of simulated $\pi^{0}$ to the total number of photon pairs satisfying the opening angle, invariant mass and leading particle selection conditions for $p p$ $(\triangle)$ and $\mathrm{Pb}-\mathrm{Pb}(\boldsymbol{\Lambda})$ collisions at $\sqrt{s}=5.5 \mathrm{~A} \mathrm{TeV}$. Only $\gamma$-jet events in the jet energy range from 20 to $100 \mathrm{GeV}$ are considered.

and rejects more than the $93 \%$ of all the uncorrelated photon pairs (Fig. 6). $\pi^{0}$ are finally identified by requiring the additional condition $120<M_{\gamma \gamma}<$ $150 \mathrm{MeV} / c^{2}$ on the invariant mass. The efficiency of the $\pi^{0}$ identification is calculated from the ratio of the number of simulated $\pi^{0}$ to the whole number of photon pairs produced in the heavy-ion event satisfying the selection conditions (Fig. 7). This ratio is between 1 and 1.2 for $\pi^{0}$ of energies greater than $5 \mathrm{GeV}$ for $p p$ collisions at $\sqrt{s}=5.5 \mathrm{TeV}$ and $10 \mathrm{GeV}$ for $\mathrm{Pb}-\mathrm{Pb}$ collisions at $\sqrt{s}=5.5 A \mathrm{TeV}$. The ratio may be larger than one because the opening angle restriction eliminates a fraction of true $\pi^{0}$ decay pairs. The combinatorial background is responsible of the fast decrease of the signal to noise ratio in $\mathrm{Pb}-\mathrm{Pb}$ collisions at low energies (Fig. 7).

$\overline{82 \% \text { at }} E_{\pi} \sim 15 \mathrm{GeV}$ and then increases again to values larger than $90 \%$ for $E_{\pi}>$ $30 \mathrm{GeV}$. 


\section{Jet reconstruction}

Jets are reconstructed starting from the seed provided by the leading particle found as described above. Particles found in a cone around the leading particle at $\left(\eta_{l}, \phi_{l}\right)$, of size $R$ defined by the equation,

$$
R=\sqrt{\left(\phi_{l}-\phi\right)^{2}+\left(\eta_{l}-\eta\right)^{2}}
$$

are assigned to a jet if their transverse momentum exceeds a given $p_{T}^{t h}$. Again, a fraction of jet particles may be lost due to the limited detector acceptance. Only jets with reconstructed energy comparable to the energy of their corresponding prompt photon are selected.

In our jet finding algorithm, two experimental configurations are considered,

(1) Charged particles are detected in the central tracking system and neutral particles in EMCal. This configuration is labeled TPC+EMCal in figures and text.

(2) Only the central tracking system is available and consequently only charged particles can be detected; this configuration is labeled TPC in figures and text.

We compare in the upper part of Fig. 8 the ratio of the measured jet transverse momentum $p_{T, j}\left(p_{T}^{\text {th }}=0.5 \mathrm{GeV} / c, R=0.3\right)$ to the energy of the corresponding prompt photon $E_{\gamma}$ for $p p$ collisions and $E_{\gamma}=40 \mathrm{GeV}$ and for both detector configurations. In the TPC+EMCal configuration, the jet energy is correctly reconstructed, and values close to the energy of the corresponding prompt photon are obtained. As the energy carried away by neutral particles can only 
be detected with EMCal, the $p_{T, j} / E_{\gamma}$ distribution does not show the expected correlation in the TPC configuration. The correlation is best observed for the highest jet energy studied. This result is independent of the cone size.

In the case of $\mathrm{Pb}-\mathrm{Pb}$ collisions, the background is large and the $p_{T, j} / E_{\gamma}$ distributions are wide and peak at values greater than unity (middle part of Fig. 8). Requiring a higher $p_{T}$ threshold than for $p p$ collisions $\left(p_{T}^{t h}=2 \mathrm{GeV} / c\right)$ to reduce the background, the distribution features (peak position and width) resemble those obtained for $p p$ collisions, at least for high-energy jets (lower part of Fig. 8). Although the width is still large for $20 \mathrm{GeV} / c$ jets, imposing a higher $p_{T}$ threshold produces a loss of essential information about the jet $(2 \mathrm{GeV} / c$ is already $10 \%$ of the jet energy). Consequently, the jet energy was calculated by requiring a $p_{T}$ threshold of $0.5 \mathrm{GeV} / c$ for $p p$ collisions and of $2 \mathrm{GeV} / c$ for $\mathrm{Pb}-\mathrm{Pb}$ collisions. Nevertheless, to construct the jet fragmentation functions, as described in next sections, all detected particles with $p_{T}^{t h}=0.5 \mathrm{GeV} / c$ inside the cone were taken into account.

Photon-jet events are well identified with the setup including EMCal when the ratio $p_{T, j} / E_{\gamma}$ is close to one. Two different values were considered for the lower $p_{T, j} / E_{\gamma}$ limits, depending on the experimental setup (with or without EMCal). The optimal selection windows (Fig. 9), depend on the energy of the reconstructed jet.

The jet reconstruction algorithm fails for jets with $p_{T, j}<10 \mathrm{GeV} / c$ due to the large fluctuations of the $p_{T, j} / E_{\gamma}$ ratio at low energies. Therefore, such jets were excluded from our investigations. 

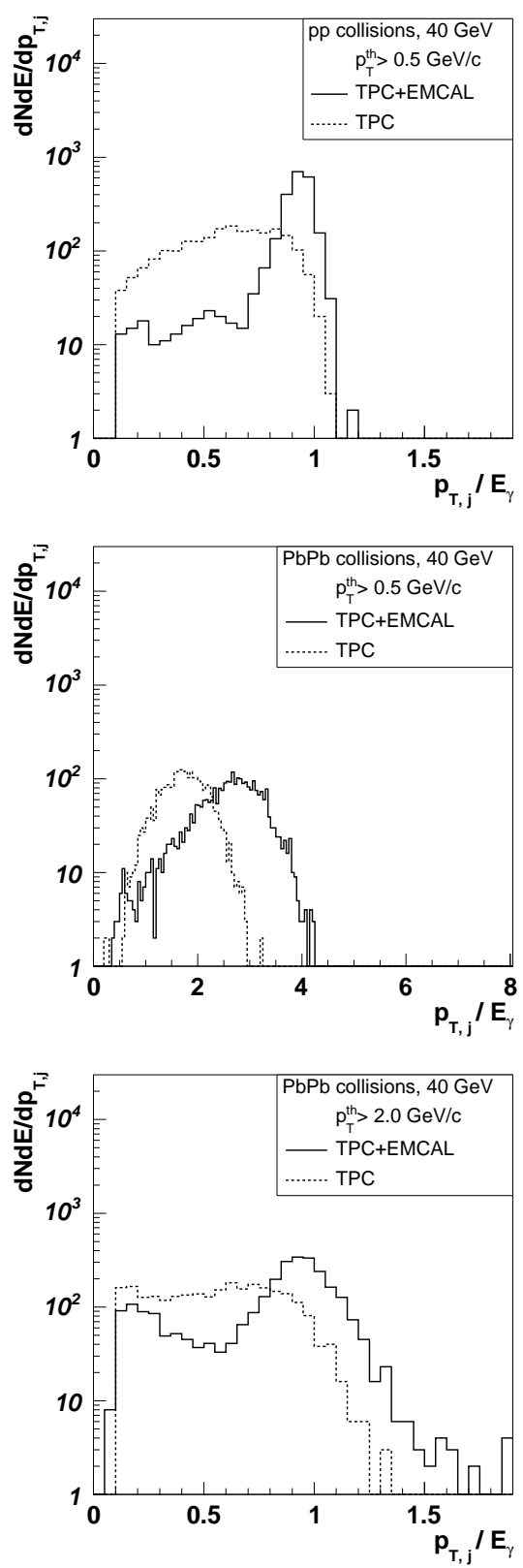

Fig. 8. Jet distribution as a function of the ratio $p_{T, j} / E_{\gamma}$ for generated $\gamma$-jet events in $p p$ collisions (top frame) and $\mathrm{Pb}-\mathrm{Pb}$ collisions (middle and bottom frames) at $\sqrt{s}=5.5 \mathrm{~A} \mathrm{TeV}$. A jet cone of $R=0.3$ and a jet particle threshold of $p_{T}^{t h}=0.5 \mathrm{GeV} / c$ are required in the top and middle frames and a jet particle threshold of $p_{T}^{t h}=2 \mathrm{GeV} / c$ is taken in the lower one. Results for the setups without EMCal (dashed line) and with EMCal (solid line) are given. 

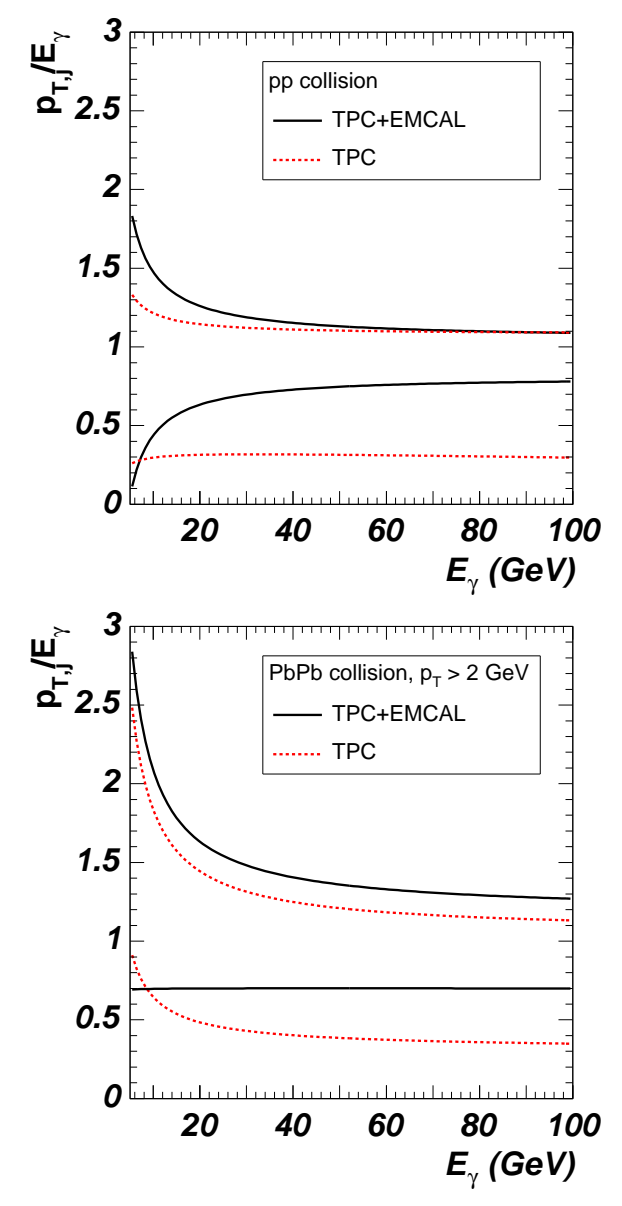

Fig. 9. Maximum (upper line) and minimum values (lower line) of the $p_{T, j} / E_{\gamma}$ ratio used to select photon-jet events in the configurations with EMCal (solid line) and without EMCal (dashed line) for $p p$ at $\sqrt{s}=5.5 \mathrm{TeV}$ (upper figure) and $\mathrm{Pb}-\mathrm{Pb}$ collisions at $\sqrt{s}=5.5 \mathrm{~A} \mathrm{TeV}$ (lower figure).

\section{$5 \quad$ Efficiency and contamination of jet selection}

The jet selection efficiency is defined as the ratio of the number of identified $\gamma$ tagged jets to the number of prompt photons detected in PHOS (Fig. 10 for $p p$ collisions and Fig. 11 for $\mathrm{Pb}-\mathrm{Pb}$ collisions). The efficiency for the configuration with EMCal is about 30\%. For the configuration without EMCal, the efficiency is higher (about 40-50\%) due to the larger acceptance of the central tracking system. To estimate the contamination level of wrongly identified $\gamma$-jets from 
jet-jet events, we applied the $\gamma$-jet algorithm also to these events. This contamination originates mainly from the decay photons contained in jets reaching PHOS, which may be misidentified as prompt photons and provide a seed for the algorithm. Indeed, the contribution of these background prompt photons can be even larger than the contribution of true prompt photons [1]. When the $\gamma$-tagging algorithm is applied to jet-jet events, a substantial fraction of misidentified prompt photons is rejected. When no prompt photon identification is performed in PHOS, only about $10 \%$ of the jet-jet events are accepted in the setup with EMCal but the value raises to $40-50 \%$ in the absence of EMCal for both $p p$ and Pb-Pb collisions (Figs. 10 and 11).

We studied the purity $\mathcal{P}$ and the contamination $\mathcal{C}$ of $\gamma$-jet identified events. The purity is defined as the fraction of true $\gamma$-jet events among the identified events and the contamination as the fraction of jet-jet events identified as $\gamma$-jet events, i.e., $\mathcal{C}=1-\mathcal{P}$. In a first step, the $\gamma$-tagging algorithm was triggered by every high- $p_{T}$ neutral signal detected in PHOS. In the case of the $\mathrm{TPC}+$ EMCal configuration, purities of about $80 \%$ and $60 \%$ for $p p$ and $\mathrm{Pb}-\mathrm{Pb}$ collisions, respectively, were obtained (Fig. 12). Without EMCal, the purity was only of $20-40 \%$. In a second step, the prompt photon identification was switched on by including a shower shape analysis (SSA) and an isolation cut method (ICM) [1]. A strong enhancement of the purity was obtained (Fig. 13). In the case of $p p$ collisions, the purity was larger than $90 \%$ for the configuration without EMCal and about $99 \%$ for the configuration with EMCal. In the case of $\mathrm{Pb}-\mathrm{Pb}$ collisions and for the configuration without EMCal the purity levels were about $80 \%$ in the whole energy range except between 30 and $50 \mathrm{GeV}$ where the purity was about $90 \%$. With EMCal the purity was higher than $90 \%$ in the entire energy range. 

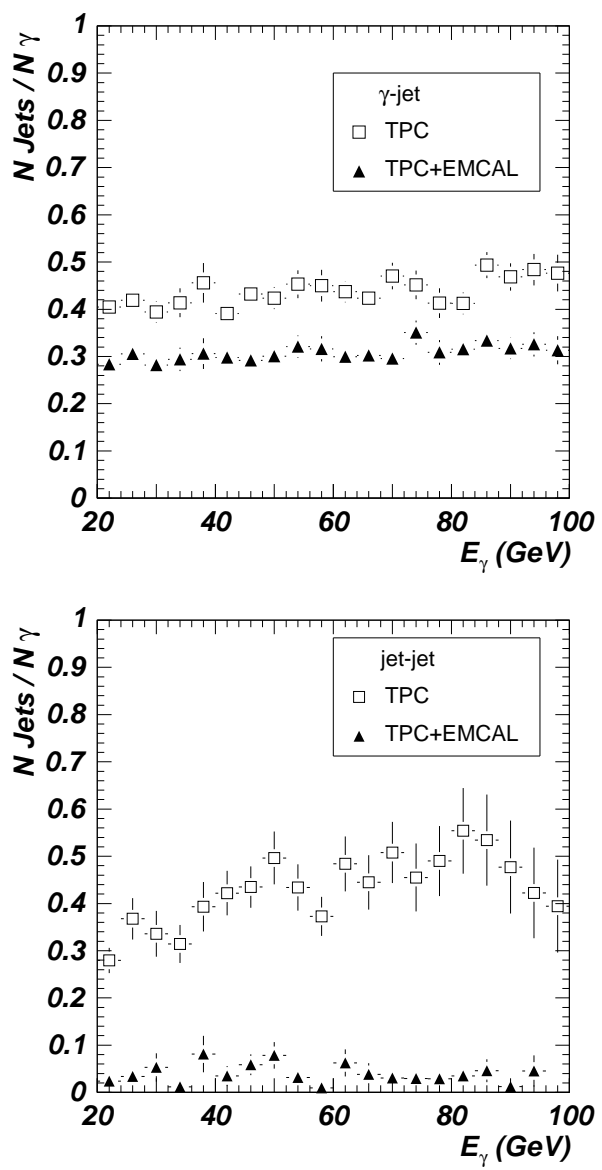

Fig. 10. Upper frame: Jet selection efficiency. Lower frame: Number of accepted jet-jet events divided by the number of $\gamma$-like particles detected in PHOS (not identified). Results for $p p$ collisions and for the setups without EMCal ( $\square$ ) and with EMCal $(\mathbf{\Lambda})$.

\section{Fragmentation functions}

A well established method to study quantitatively the interaction of jets with the medium is to investigate the redistribution of fragmentation hadrons in phase space [4], i.e., to measure the jet fragmentation function. The experimental fragmentation function is the distribution of charged hadrons within jets as a function of the variable $z$, defined for hard processes with a $\gamma$-jet pair in the final state as $z=p_{T} / E_{\gamma}$. The statistics for the fragmentation function 

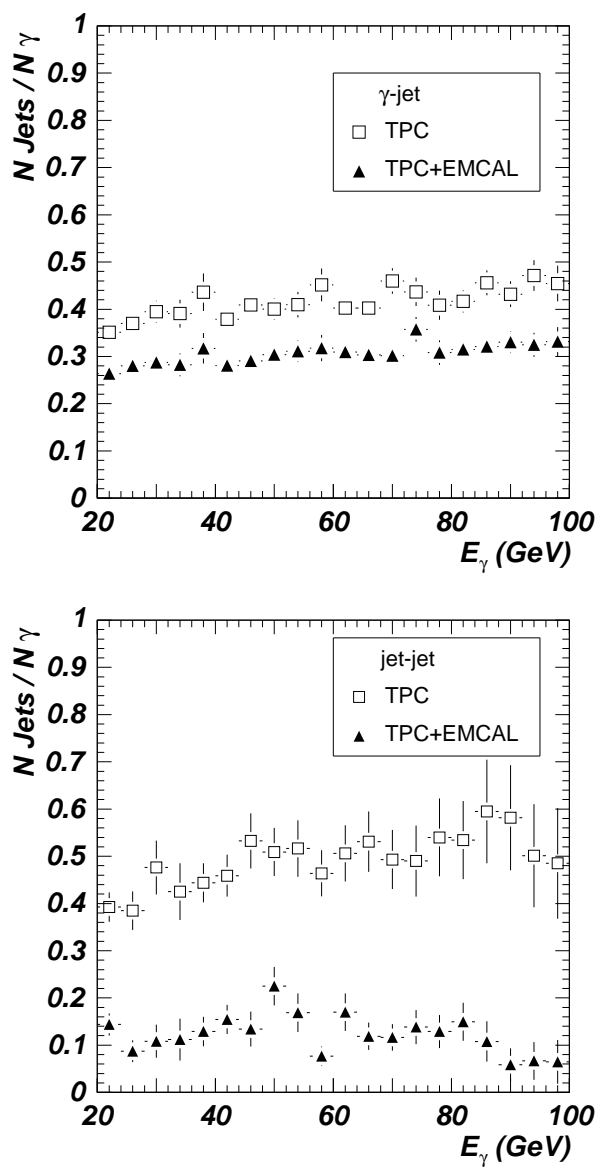

Fig. 11. Upper frame: Jet selection efficiency. Lower frame: Number of accepted jet-jet events divided by the number of $\gamma$-like particles detected in PHOS (not identified). Results for $\mathrm{Pb}-\mathrm{Pb}$ collisions at $\sqrt{s}=5.5 A \mathrm{TeV}$ and for the setups without EMCal ( $\square)$ and with EMCal (ム).

measurement during a standard year of LHC running was estimated for both $p p$ and $\mathrm{Pb}-\mathrm{Pb}$ collisions at $\sqrt{s}=5.5 A \mathrm{TeV}$ (Figs. 14 and 15). These fragmentation functions were constructed from $\gamma$-jet events and misidentified jet-jet events by integrating all events with identified prompt photon energy larger than $20 \mathrm{GeV} / c$. The following conclusions are obtained:

(1) In the case of $p p$ collisions, in the absence of EMCal and without prompt photon identification, the contamination due to misidentified jet-jet events dominates the true $\gamma$-jet contribution. In the setup with EMCal the con- 
$p p$ collisions

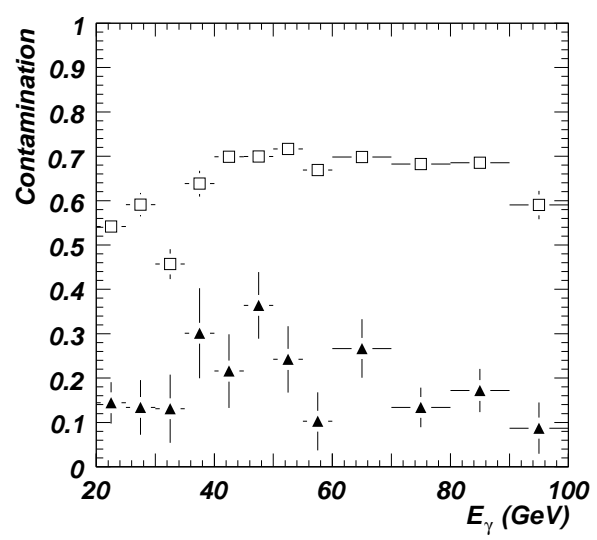

$\mathrm{Pb}-\mathrm{Pb}$ collisions

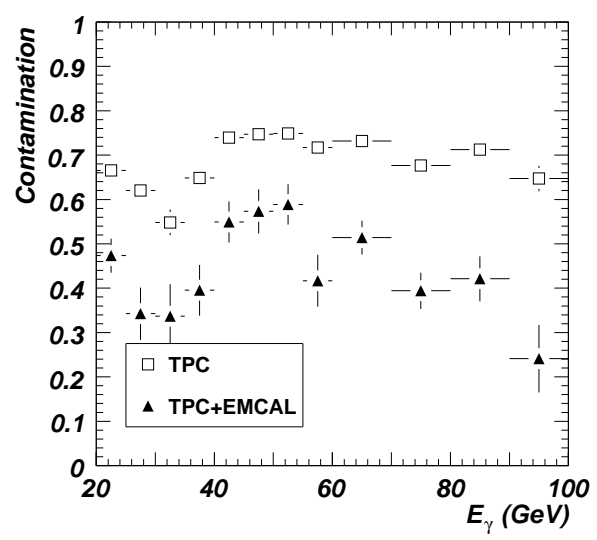

Fig. 12. $\gamma$-tagging contamination without prompt photon identification in PHOS, i.e., a high- $p_{T}$ neutral signal triggers the jet finding algorithm. Upper (lower) frame corresponds to $p p(\mathrm{~Pb}-\mathrm{Pb})$ collisions at $\sqrt{s}=5.5 A \mathrm{TeV}$. Results shown for the setups without EMCal ( $\square)$ and with EMCal (ム).

tamination is suppressed, leading to a signal to background ratio close to 4. If prompt photons in PHOS are identified by medium purity SSA and ICMS (an isolation cut method with a threshold on the total $p_{T}$ sum) [1], the contamination of misidentified jet-jet events is largely reduced, leading to a signal to background ratio of about 20 in the configuration without EMCal and near to $100 \%$ background rejection for the setup with EMCal. This reduction of background is accompanied by a reduction of 
$p p$ collisions

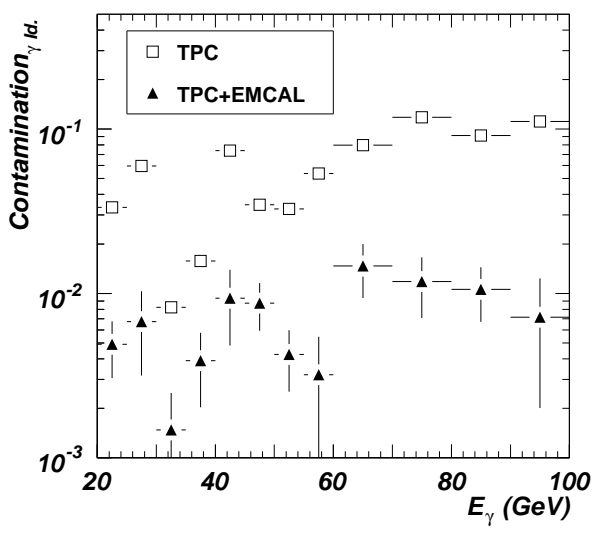

$\mathrm{Pb}-\mathrm{Pb}$ collisions

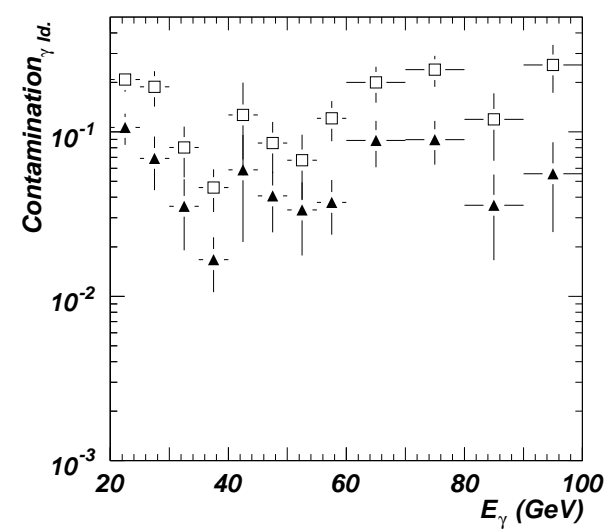

Fig. 13. $\gamma$-tagging contamination with prompt photon identification in PHOS (SSA+ICM, see definitions in Refs. $[1,6]$ ). Upper (lower) frame corresponds to $p p(\mathrm{~Pb}-\mathrm{Pb})$ collisions at $\sqrt{s}=5.5 \mathrm{~A} \mathrm{TeV}$. Results shown for the setups without EMCal ( $\square)$ and with EMCal $(\mathbf{\Lambda})$.

the $\gamma$-jet statistics of only $10 \%$.

(2) In the case of $\mathrm{Pb}-\mathrm{Pb}$ collisions, the measured fragmentation function presents a peak at low $z$, an artifact due to low- $p_{T}$ charged hadrons coming from the heavy-ion underlying event. This peak can be statistically subtracted by calculating the contribution outside the cone defined by the leading particle. This contribution is estimated by constructing a pseudofragmentation function with the particles inside a cone (with the same $R$ 
$p p$ collisions

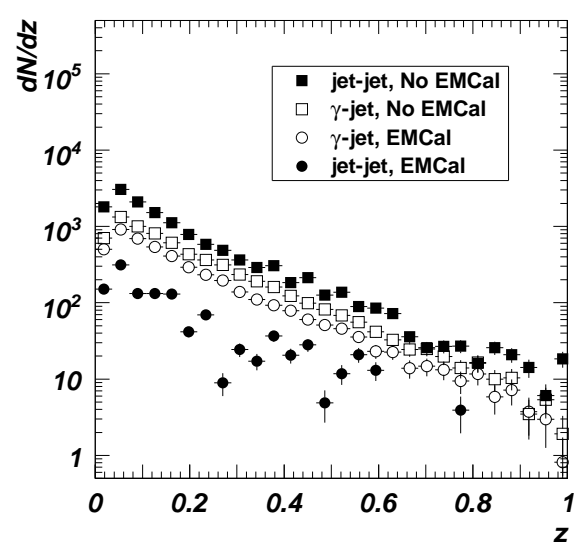

$\mathrm{Pb}-\mathrm{Pb}$ collisions

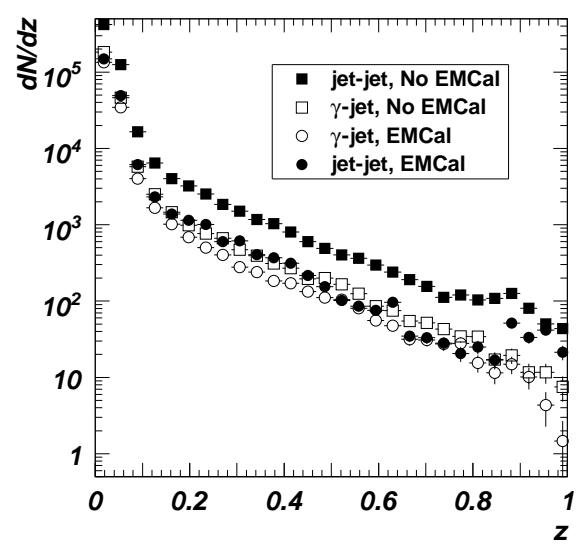

Fig. 14. Fragmentation function for $\gamma$-jet and jet-jet events with photon energy larger than $20 \mathrm{GeV}$ for $p p$ (upper frame) and $\mathrm{Pb}-\mathrm{Pb}$ (lower frame) collisions at $\sqrt{s}=5.5 A \mathrm{TeV}$. Neither prompt photon identification in PHOS nor heavy-ion environment background subtraction are done.

as the jet cone) centered at $\left(\phi_{\gamma}, \eta_{\text {leading }}\right)$ which for $\gamma$-jet events is a region populated only by particles coming from the HIJING event with no contribution from the jet. Prompt photon identification by medium purity SSA and ICM was required to reduce the contamination of wrongly identified jet-jet events to an acceptable level. The final signal to background ratio obtained is about 4 in the case without EMCal and rises up to 10 with EMCal. The requirement of prompt photon identification reduces 
$p p$ collisions

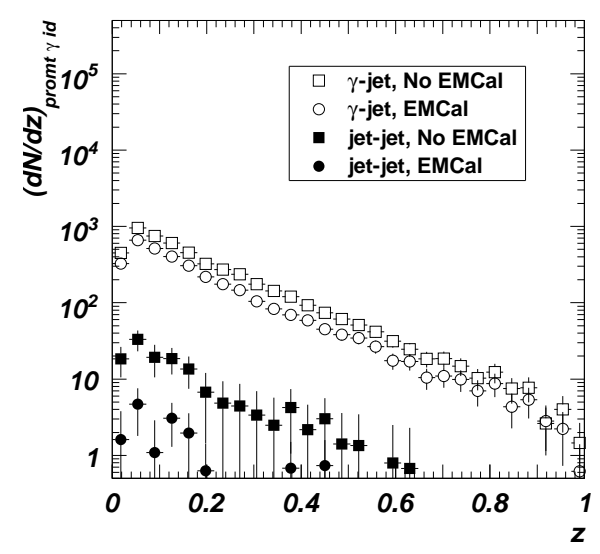

$\mathrm{Pb}-\mathrm{Pb}$ collisions

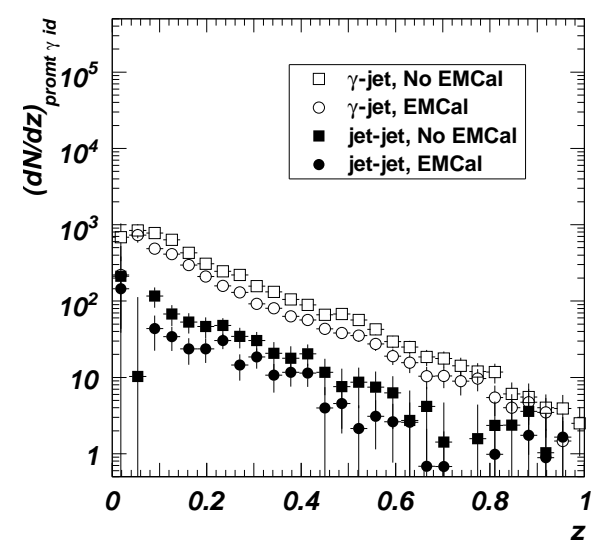

Fig. 15. Fragmentation function for $\gamma$-jet and jet-jet events with photon energy larger than $20 \mathrm{GeV}$ for $p p$ (upper frame) and $\mathrm{Pb}-\mathrm{Pb}$ (lower frame) collisions at $\sqrt{s}=5.5 A \mathrm{TeV}$. Prompt photons are identified in PHOS by medium purity SSA and ICM ( $p p$ collisions) or ICMS (Pb-Pb collisions), and the heavy-ion background is statistically subtracted (see Refs. $[1,6]$ for details on identification definitions).

the statistics of $\gamma$-jet events by about $60 \%$.

The fragmentation functions were calculated as the sum of the identified $\gamma$-jet events and jet-jet background events scaled by the corresponding cross section (Figs. 16 and 17). Statistical errors were calculated from the statistics that can be accumulated in a standard LHC year of running. Systematic errors reflect the amount of contamination. For $\mathrm{Pb}-\mathrm{Pb}$ collision, in the range $0.1<$ 


\section{TPC+EMCal}

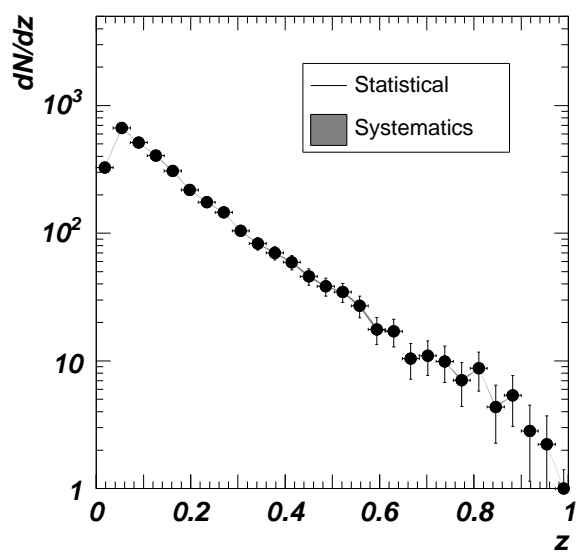

$\mathrm{TPC}$

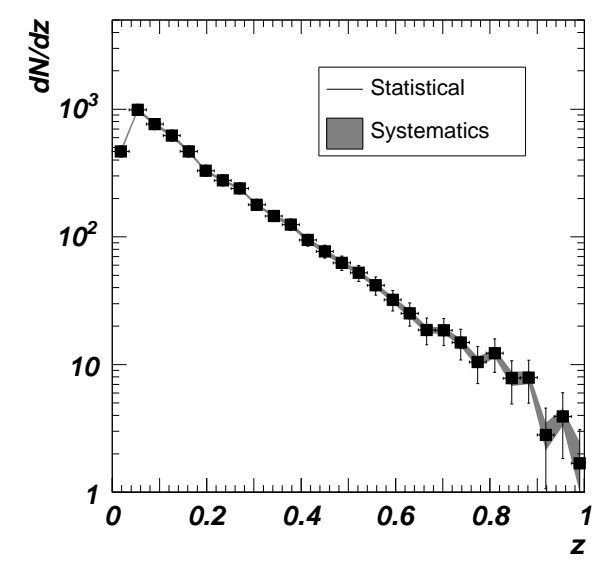

Fig. 16. Fragmentation function for $\gamma$-tagged jets $(\gamma$-jet + jet-jet events after prompt photon identification) with photon energy larger than $20 \mathrm{GeV}$ for a whole ALICE year, detected in the central tracking system and EMCal (upper frame) and in the central tracking system alone (lower frame), for $p p$ collisions. The shaded regions represent the systematic error due to jet-jet contamination.

$z<0.5$, systematic and statistical errors are of the same order, both with and without EMCal. Statistical errors outside this $z$ range are too large to measure medium modifications effects. The inclusion of EMCal reduces the systematic errors by a factor of about 5 in $p p$ collisions and about 2 in $\mathrm{Pb}-\mathrm{Pb}$ collisions. When high $p_{T} \pi^{0}$ suppression as observed at RHIC $[12,15,14,13]$ is accounted for, systematic errors are additionally reduced by a factor 5 , 


\section{TPC+EMCal}

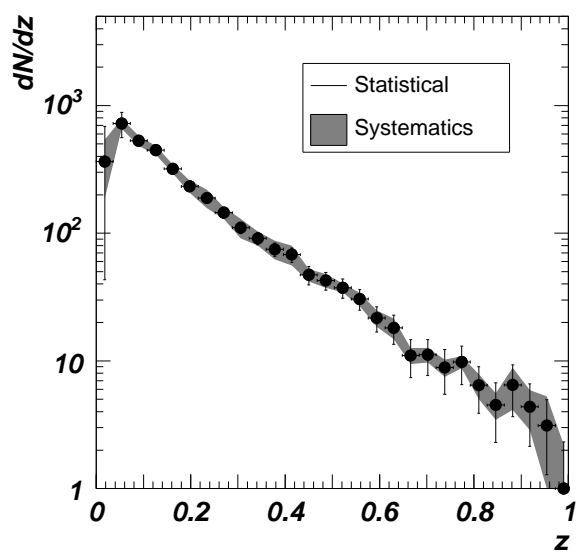

$\mathrm{TPC}$

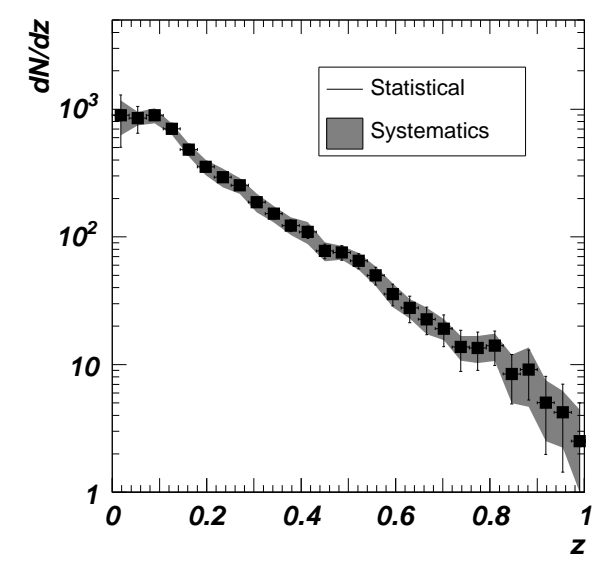

Fig. 17. Fragmentation function for $\gamma$-tagged jets $(\gamma$-jet + jet-jet events after prompt photon identification) with photon energy larger than $20 \mathrm{GeV}$ for a whole ALICE year, detected in the central tracking system and EMCal (upper frame) and in the central tracking system alone (lower frame), for $\mathrm{Pb}-\mathrm{Pb}$ collisions at $\sqrt{s}=5.5 \mathrm{~A} \mathrm{TeV}$. The shaded regions represent the systematic error due to jet-jet contamination.

becoming thus systematically smaller than the statistical errors, both with and without EMCal.

The sensitivity of photon-tagged jet fragmentation functions to nuclear medium modifications can be estimated from the nuclear modification factor $R_{F F}$. This factor is defined as the ratio of the fragmentation functions measured in $A A$ 


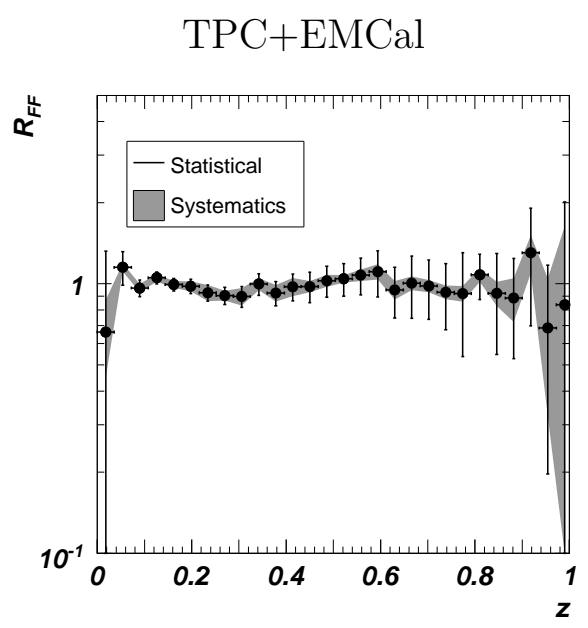

TPC

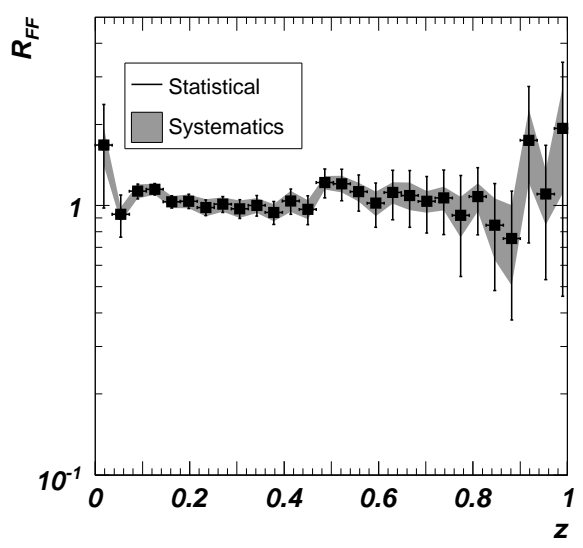

Fig. 18. Ratio of the fragmentation functions of $\gamma$-tagged jets with photon energy larger than $20 \mathrm{GeV}$ for $\mathrm{Pb}-\mathrm{Pb}$ collisions at $\sqrt{s}=5.5 \mathrm{~A} \mathrm{TeV}$ scaled by the number of binary collisions [1] to $p p$ collisions at $\sqrt{s}=5.5 \mathrm{TeV}$ detected in the central tracking system and EMCal (upper frame) or in the central tracking system alone (lower frame). The shaded region represents the systematic error due to contamination from jet-jet events.

and $p p$ collisions scaled to the number of binary $N N$ collisions, with both fragmentation functions calculated for the same beam luminosity and running time. $R_{F F}$ should be equal to unity in the absence of nuclear effects. As no medium modification effect was included in our simulations, we obtain indeed a value close to one over the entire $z$ range (Fig. 18). The statistical 


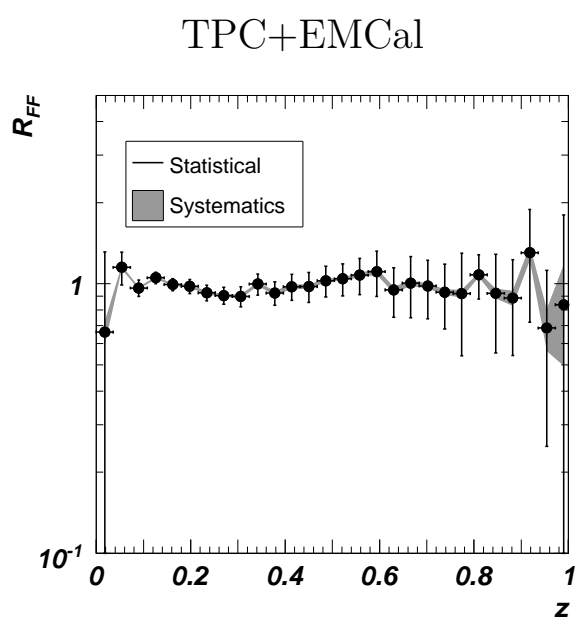

TPC

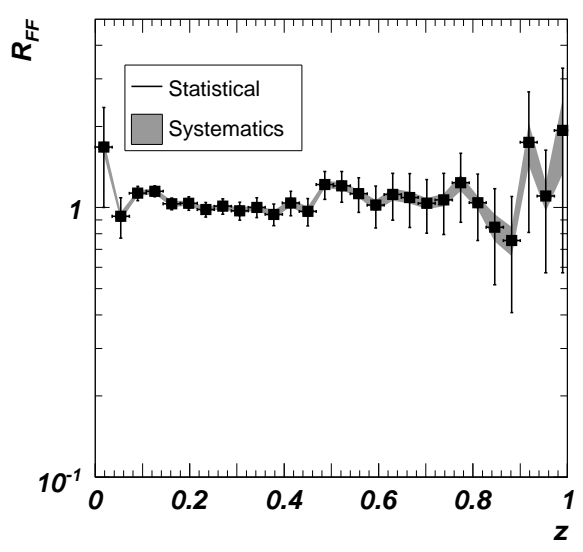

Fig. 19. Ratio of the fragmentation functions of $\gamma$-tagged jets with photon energy larger than $20 \mathrm{GeV}$ for $\mathrm{Pb}-\mathrm{Pb}$ collisions at $\sqrt{s}=5.5 \mathrm{~A} \mathrm{TeV}$ scaled by the number of binary collisions [1] to $p p$ collisions at $\sqrt{s}=5.5 \mathrm{TeV}$ detected in the central tracking system and EMCal (upper frame) or in the central tracking system alone (lower frame). The shaded region represents the systematic error due to the contamination from jet-jet events. Background is quenched a factor 5 like at RHIC.

and systematic errors indicate that variations of $R_{F F}$ larger than $5 \%$ could be measured for both setups in the range $0.1<z<0.5$. If $\pi^{0}$ are quenched by a factor of 5, as observed at RHIC, the systematic error would be smaller than $5 \%$ for both setups, as displayed in Fig. 19. However, there is no possibility to measure the nuclear modification factor with an accuracy better than $5 \%$ 
due to limited $\gamma$-jet statistics expected in one year of data taking.

We may still consider another measurement approach in which prompt photons are detected by EMCal and jets are only measured by the central tracking system ${ }^{5}$. In this setup, the prompt photon counting rate would be enhanced by a factor of 7 and consequently the statistical errors would be reduced by a factor 2.6 (figures obtained by assuming the responses of PHOS and EMCal identical). This reduction of statistical errors may allow to measure medium modification effects over a wider $z$ range.

\section{Conclusions}

We have developed an algorithm to identify $\gamma$-jet events generated in $p p$ and $\mathrm{Pb}-\mathrm{Pb}$ collisions at $\sqrt{s}=5.5 \mathrm{~A} \mathrm{TeV}$ with the LHC detector ALICE. The $\gamma$-jet events are identified by selecting a prompt photon in PHOS and searching for the leading particle in the opposite direction inside the ALICE central tracking system. Two different setups, with and without the electromagnetic calorimeter EMCal, are considered. Jets are reconstructed by an algorithm in which the leading particle is used as a seed. The reconstructed jet is correlated to the photon if a number of conditions are fulfilled. The efficiency of identifying $\gamma$-jet events is mainly determined by the acceptance of the central tracking system and EMCal. For jets of energy larger than $20 \mathrm{GeV}$, this efficiency is found to be from around $40 \%$ to $50 \%$ with increasing photon energy without EMCal, and, due to the smaller EMCal acceptance, about $30 \%$ with EMCal. A large contribution to the $\gamma$-jet event background comes from

5 It is not advisable to use PHOS as a detector of jet neutral particles due to its reduced acceptance. 
$\pi^{o}$ decay photons misidentified in PHOS as prompt photons. With our $\gamma$-jet tagging method in combination with isolation cut analysis for prompt photon identification, the misidentification of jet-jet events as $\gamma$-jet events in $p p$ collisions is of the order of $5 \%$ without EMCal and less than $1 \%$ with EMCal, and in $\mathrm{Pb}-\mathrm{Pb}$ collisions is of the order of $20 \%$ without EMCal and around $10 \%$ with EMCal. We obtain from our simulations that fragmentation functions could be measured with sufficient accuracy to obtain the nuclear modification factor $R_{F F}$ with errors low enough to probe medium modifications. As a main conclusion, we find that nuclear medium modifications could be measured if they produce variations of $R_{F F}$ larger than $5 \%$ in the region $0.1<z<0.5$.

\section{Acknowledgment}

This work has been supported in part by the Spanish DGICYT under contract FPA2003-07581-C02-01. One of us (G. C.) thanks Ministerio de Educación y Ciencia for a PhD fellowship contract FP200-5452 and the Marie Curie EU program for the "Training Site" contract HPMT-CT-2001-00346. Also, we thank the support of the INTAS grant 03-52-5747.

\section{References}

[1] G. Conesa et al., Nucl. Instr. and Meth. A580 (2007) 1446.

[2] A. Accardi et al, hep-ph/0310274 (2004).

[3] J.D. Bjorken, FERMILAB-PUB-82-059-THY (1982).

[4] C. A. Salgado and U.A. Wiedemann, Phys. Rev. Lett. 93 (2004) 42301. 
[5] F. Arleo et al., JHEP 11 (2004) 009.

[6] ALICE Collaboration et al., Physics performace report, Volume II, J. Phys. G: Nucl. Part. Phys. 32 (2006) 1295-2040

[7] T. Sjostrand et al., Comput. Phys. Commun. 135 (2001) 238-259.

[8] T. Sjostrand et al., PYTHIA 6.2: Physics and manual, hep-ph/0108264 (2001).

[9] M. Gyulassy and X.N. Wang, Comput. Phys. Commun. 83 (1994) 307-331.

[10] ALICE Collaboration, J. Phys. G: Nucl. Part. Phys. 30 (2004)1517-1763.

[11] T. Cormier et al., Eur Phys J, C 34, (2004) s01, s333-345

[12] J. Adams et al., the STAR collaboration, Nucl. Phys. A757 (2005) 102.

[13] K. Adcox et al., thePHENIX collaboration, Nucl. Phys. A757 (2005) 184.

[14] I. Arsene et al., the BRAHMS collaboration, Nucl. Phys. A757 (2005) 1.

[15] M. B. Tonjes et al., PHOBOS collaboration, Nucl. Phys. A757 (2005) 61. 